\title{
ON 1-VERTEX BIMAGIC VERTEX LABELING
}

\author{
J. BASKAR BABUJEE AND S. BABITHA
}

\begin{abstract}
A 1-vertex magic vertex labeling of a graph $G$ with $p$ vertices is defined as a bijection $f$ from the vertices to the integers $1,2, \ldots, p$ with the property that there is a constant $k$ such that at any vertex $x, \sum_{y \in N(x)} f(y)=k$, where $N(x)$ is the set of vertices adjacent to $x$. In this paper we introduce 1-vertex bimagic vertex labeling of a graph $G$ and obtain the necessary condition for a graph to be 1-vertex bimagic. We exhibit the same type of labeling for some class of graphs and give some general results.
\end{abstract}

\section{Introduction}

A labeling of a graph is assigning labels to the vertices, edges or both vertices and edges. In most applications labels are positive (or nonnegative) integers, though in general real numbers could be used. For various types of graph labeling one can refer the survey of graph labeling by J.A. Gallian [3]. In 1963, Sedláĉek [7] introduced the magic labeling for a graph $G=G(V, E)$ which is defined as a bijection $f$ from $E$ to a set of positive integers such that

(i) $f\left(e_{i}\right) \neq f\left(e_{j}\right)$ for all distinct $e_{i}, e_{j} \in E$, and

(ii) $\sum_{e \in N_{E}(x)} f(e)$ is the same for every $x \in V$, where $N_{E}(x)$ is the set of edges incident to $x$.

MacDougall, Miller, Slamin and Wallis [4] introduced the notion of a vertex-magic total labeling in 1999. For a graph $G(V, E)$ a bijective mapping $f$ from $V \cup E$ to the set $\{1,2, \ldots,|V \cup E|\}$ is a vertex-magic total labeling if there is a constant $k$, called the magic constant, such that for every vertex $v, f(v)+\sum f(v u)=k$ where the sum is over all vertices $u$ adjacent to $v$ (some authors use the term "vertex-magic" for this concept). In [1, 2], edge bimagic total labeling was introduced by J. Baskar Babujee. A graph $G(p, q)$ with $p$ vertices and $q$ edges is called edge bimagic total if there exists a bijection $f: V \cup E \rightarrow\{1,2, \ldots, p+q\}$ such that for any edge $u v \in E$, we have two constants $k_{1}$ and $k_{2}$ with $f(u)+f(v)+f(u v)=k_{1}$ or $k_{2}$.

Received September 13, 2011, accepted March 11, 2014. 2010 Mathematics Subject Classification. 05C78.

Key words and phrases. Labeling, bijection, magic, bimagic, regular graph. Corresponding author: J. Baskar Babujee . 
In [5] Mirka Miller et al. defined the 1-vertex-magic vertex labeling of a graph with $p$ vertices defined as a bijection $f$ taking the vertices to the integers $1,2, \ldots, p$ with the property that there is a constant $k$ such that at any vertex $x, \sum_{y \in N(x)} f(y)=k$, where $N(x)$ is the set of vertices adjacent to $x$ (that is, distance 1 from $x$ ).

A necessary condition for the existence of a 1-vertex magic vertex labeling is given in [5] as follows:

If $f$ is 1-vertex magic vertex labeling then $\sum_{x \in V} d(x) f(x)=k p$ where $d(x)$ is the degree of vertex $x$.

A 1-vertex-magic vertex labeling is the same as sigma labeling or $\Sigma$-labeling defined by Vilfred [11]. It becomes interesting when we arrive with 1-vertex magic vertex labeling summing to exactly two distinct constants say $k_{1}$ or $k_{2}$. This motivates us to work in 1-vertex bimagic vertex labeling. This paper is organized as follows. In section 2, we introduce a 1vertex bimagic vertex labeling and which classes of graphs are and are not 1-vertex bimagic. In section 3, we prove a 1-vertex bimagic vertex labeling for complete symmetric multipartite graph. In section 4 , we prove some general results on regular or bi-regular1-vertex bimagicgraphs. In section 5, we conclude this paper with an open problem.

\section{1-Vertex Bimagic Vertex Labeling}

Definition 2.1. A bijective labeling $f: V(G) \rightarrow\{1,2, \ldots, p\}$ is called a 1-vertex bimagic vertex labeling if for each vertex $u \in V(G)$, the sum of all $f(v)$ such that $v$ is adjacent to $u$ is either $k_{1}$ or $k_{2}$ (i.e) for all $u \in V(G), \sum_{u v \in E(G)} f(\nu)=k_{1}$ or $k_{2}$ Where $k_{1}$ and $k_{2}$ are distinct constants. A graph which has a 1-vertex bimagic vertex labeling is called a 1-vertex bimagic graph.

Definition 2.2. A bijective labeling $f: V(G) \rightarrow\{1,3, \ldots, 2 p-1\}$ is called a odd 1-vertex bimagic vertex labeling if for each vertex $u \in V(G)$, the sum of all $f(v)$ such that $v$ is adjacent to $u$ is either $k_{1}$ or $k_{2}$ (i.e) for all $u \in V(G), \sum_{u v \in E(G)} f(v)=k_{1}$ or $k_{2}$ Where $k_{1}$ and $k_{2}$ are distinct constants.

Definition 2.3. A bijective labeling $f: V(G) \rightarrow\{0,2, \ldots, 2(p-1)\}$ is called a even 1-vertex bimagic vertex labeling if for each vertex $u \in V(G)$, the sum of all $f(v)$ such that $v$ is adjacent to $u$ is either $k_{1}$ or $k_{2}$ (i.e) for all $u \in V(G), \sum_{u v \in E(G)} f(\nu)=k_{1}$ or $k_{2}$ Where $k_{1}$ and $k_{2}$ are distinct constants.

Theorem 2.1. A necessary condition for the existence of a 1-vertex bimagic vertex labeling $f$ of a graph $G$ is

$$
\sum_{x \in V} d(x) f(x)=k_{1} p_{1}+k_{2} p_{2}
$$


where $d(x)$ is the degree of vertex $x$ and $p_{1}, p_{2}$ are the number of vertices with common count $k_{1}$ and $k_{2}$ respectively.

Proof. Let $V=V_{1} \cup V_{2}$ and $\left|V_{1}\right|=p_{1},\left|V_{2}\right|=p_{2}=p-p_{1}$ where $p_{1}$ and $p_{2}$ the number of vertices with common count $k_{1}$ and $k_{2}$ respectively. Consider L.H.S of (2.1),

$$
\sum_{x \in V} d(x) f(x)=\sum_{x \in V_{1}} d(x) f(x)+\sum_{x \in V_{2}} d(x) f(x)
$$

Consider the two sub graphs $G_{1}$ and $G_{2}$ of $G$ with vertex sets $V_{1}$ and $V_{2}$ respectively. Applying the necessary condition for the existence of a 1-vertex magic vertex labeling given in [5] mentioned in introduction of our paper, (2.2) becomes

$$
\sum_{x \in V} d(x) f(x)=k_{1} p_{1}+k_{2} p_{2}
$$

Theorem 2.2. If $G$ has a 1-vertex magic vertex labeling and $G \neq C_{4}$, then $G+K_{1}$ admits a 1vertex bimagic vertex labeling.

Proof. If $G$ is cycle $C_{4}$ then $C_{4}+K_{1}$ is 1-vertex magic. Let $G(p, q)$ has a 1-vertex magic vertex labeling then there exist a function $f: V \rightarrow\{1,2, \ldots, p\}$ such that for every vertex $u, \sum_{u \nu \in E(G)} f(\nu)=$ $r$. Now we define the new graph called $G_{1}=G+K_{1}$ with vertex set $V_{1}\left(G_{1}\right)=V(G) \cup\{x\}$ and $E_{1}\left(G_{1}\right)=E(G) \cup\left\{x v_{i}: 1 \leq i\right.$ leqp $\}$. Consider the bijective function $g: V_{1} \rightarrow\{1,2, \ldots, p, p+1\}$ defined by $g\left(v_{i}\right)=f\left(v_{i}\right) ; 1 \leq i \leq p$ or $v_{i} \in V(G)$ $g(x)=p+1$.

Since the graph $G$ is already 1 -vertex magic vertex labeling, for every $u \in V_{1}(G)$,

$$
\begin{aligned}
\sum_{u v \in E_{1}\left(G_{1}\right)} g(v) & =\sum_{u v \in E(G)} f(\nu)+g(x) \\
& =r+p+1=k_{1} \text { (say) }
\end{aligned}
$$

For the newly added vertex $x$,

$$
\sum_{\nu \in N(x)} g(v)=\sum_{i=1}^{p} g\left(v_{i}\right)=\sum_{i=1}^{p} f\left(v_{i}\right)=1+2+3+\cdots+p=\frac{p(p+1)}{2}=k_{2} \text { (say) }
$$

This proves that $G+K_{1}$ admits a 1-vertex bimagic vertex labeling.

Theorem 2.3. The path graph $P_{n}, n>3$ is not a 1-vertex bimagic.

Proof. If $n \leq 3, P_{n}$ is a 1-vertex bimagic. Consider the path $P_{n}, n>3$ with vertex set $\left\{v_{1}, v_{2}, \ldots, v_{n}\right\}$ and edge set $\left\{v_{i} v_{i+1}: 1 \leq i \leq n-1\right\}$. If we arrange the labels $\left\{a_{1}, a_{2}, \ldots, a_{n}\right\}$ for the vertices 
$\left\{v_{1}, v_{2}, \ldots, v_{n}\right\}$ by permutation, the common count of $v_{1}$ is $a_{2}=k_{1}$ (say) and $v_{n}$ is $a_{n-1}=k_{2}$ (say). For all other vertices from $\left\{v_{2}, \ldots, v_{n-1}\right\}$ the common count is $\left\{a_{1}+a_{3}, a_{2}+a_{4}, \ldots, a_{n-2}+\right.$ $a_{n}$ \} respectively. Since the function $f$ is bijective, (all the vertices having distinct labeling) these $n-2$ counts cannot be either $k_{1}$ or $k_{2}$. Hence the path graph $P_{n}$ is not a 1 -vertex bimagic.

Theorem 2.4. The cycle graph $C_{n}$ is a 1-vertex bimagic if and only if $n=4$.

Proof. Consider the cycle $C_{n}(n \geq 5)$ with vertex set $\left\{v_{1}, v_{2}, \ldots, v_{n}\right\}$ and edge set $\left\{v_{i} v_{i+1}: 1 \leq\right.$ $i \leq n-1\} \cup\left\{v_{n} v_{1}\right\}$. If we arrange the labels $\left\{a_{1}, a_{2}, \ldots, a_{n}\right\}$ for the vertices $\left\{v_{1}, v_{2}, \ldots, v_{n}\right\}$ by permutation, the common count of $v_{2}$ is $a_{1}+a_{3}=k_{1}$ (say) and $v_{n}$ is $a_{n-1}+a_{1}=k_{2}$ (say). For all other vertices $\left\{v_{1}, v_{3}, \ldots, v_{n-1}\right\}$ the common count is $\left\{a_{1}+a_{n}, a_{2}+a_{4}, \ldots, a_{n-2}+a_{n}\right\}$ respectively. Since the function $f$ is bijective, (all the vertices having distinct labeling) these $n-2$ counts cannot be either $k_{1}$ or $k_{2}$. Hence the cycle $C_{n}(n \geq 5)$ is not a 1 -vertex bimagic.

Conversely, Consider the cycle $C_{n}$ with $n=4$. The vertices of the cycle $C_{4}$ are $v_{1}, v_{2}, v_{3}, v_{4}$. Let the function $f: V(G) \rightarrow\{1,2,3,4\}$ be defined as $f\left(v_{i}\right)=i$. Consider the common count of the labels of $v_{1}$ and $v_{3}$, we have $\sum_{v_{3}} f(u)=f(G)=f\left(v_{2}\right)+f\left(v_{4}\right)=6$ and for the common count of the labels of $v_{2}$ and $v_{4}$, we have $\sum_{v_{3}} \sum_{\in E(G)} f(u)=f\left(v_{1}\right)+f\left(v_{3}\right)=4$. Thus for the cycle $C_{4}$, we have two constants 6 and 4 . Hence the cycle $C_{n}$ is a 1-vertex bimagic when $n=4$.

Theorem 2.5. The complete bipartite graph $K_{m . n}$ has a 1-vertex bimagic vertex labeling.

Proof. Let $G(V, E)$ be a complete bipartite graph with vertex set $V(G)=\left\{v_{1}, v_{2}, \ldots, v_{m}, u_{1}, u_{2}, \ldots\right.$, $\left.u_{n}\right\}$ and edge set be $E(G)=\left\{v_{i} u_{j}: 1 \leq i \leq m, 1 \leq i \leq n\right\}$. Define the function $f: V(G) \ldots\{1,2, \ldots$, $m+n\}$ as follows

If $m \leq n$,

$f\left(v_{i}\right)=i$ for $1 \leq i \leq m$,

$f\left(u_{j}\right)=m+j$ for $1 \leq j \leq n$

If $m>n$,

$f\left(v_{i}\right)=i$ for $1 \leq i \leq n$,

$f\left(u_{j}\right)=n+j$ for $1 \leq j \leq m$

If $m \leq n$, for any vertex $v_{i} \in V(G)$,

$$
\sum_{v_{i}} f(u)=\sum_{j=1}^{n} u_{j}=\sum_{j=1}^{n} m+j=n m+(1+2+\cdots+n)=n m+\frac{n(n+1)}{2}=k_{1}
$$

For any vertex $u_{j} \in V(G)$,

$$
\sum_{u_{i} u \in E(G)} f(u)=\sum_{i=1}^{m} v_{i}=\sum_{i=1}^{m} i=1+2+\cdots+m=\frac{m(m+1)}{2}=k_{2}
$$


Thus for any vertex $v \in V(G)$ we have two constants $k_{1}$ or $k_{2}$.

Similarly for $m>n$, we get the two constants $k_{1}$ and $k_{2}$ vice versa. Which proves that complete bipartite graph $K_{m . n}$ has a 1-vertex-bimagic vertex labeling.

Definition 2.4. A graph $G^{o}\left(P_{n}\right)$ is obtained from a path $P_{n}$ by introducing new edges between any two vertices if they are at odd distance.

$G^{o}\left(P_{n}\right)$ has $n$ vertices and $\left(\frac{n^{2}-1}{4}\right)$ edges if $n$ is odd and $\left(\frac{n^{2}}{4}\right)$ edges if $n$ is even.

Also $G^{o}\left(P_{n}\right)$ is $\left(\frac{n-1}{2}, \frac{n+1}{2}\right)$ bi-regular when $n$ is odd and $\left(\frac{n}{2}\right)$ regular when $n$ is even.

The 1-vertex-bimagic vertex labeling for $G^{o}\left(P_{9}\right)$ and $G^{o}\left(P_{10}\right)$ is shown below.

\section{Example 2.1.}
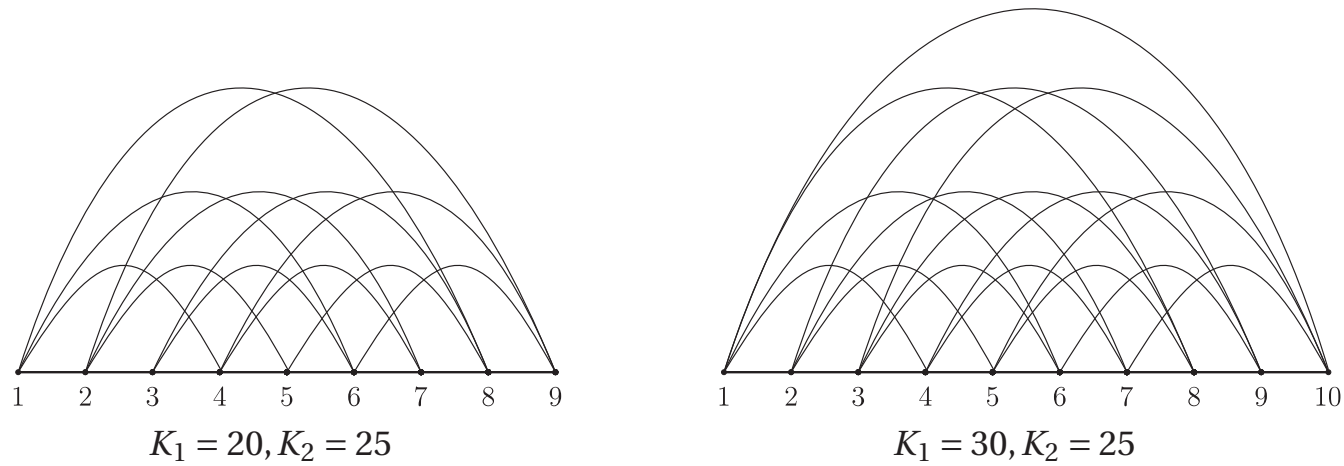

Figure 1: 1-vertex bimagic vertex labeling for $G^{o}\left(P_{9}\right)$ and $G^{o}\left(P_{10}\right)$.

Theorem 2.6. The graph $G^{o}\left(P_{n}\right), n \geq 3$ has 1-vertex bimagic vertex labeling.

Proof. Consider the graph $G^{o}\left(P_{n}\right)$. We prove this theorem in two cases

Case 1: When $n$ is odd

The vertex set and edge set be defined as $V(G)=\left\{v_{1}, v_{2}, \ldots, v_{n}\right\}$. As per the definition of $G^{o}\left(P_{n}\right)$ we construct the edge set with respect to the index of the vertices as follows $E(G)=$ $\left\{v_{i} v_{2 j}: 1 \leq j \leq \frac{n-1}{2}\right.$ and for $i$ is odd $\} \cup\left\{v_{i} v_{2 j-1}: 1 \leq j \leq \frac{n+1}{2}\right.$ and for $i$ is even $\}$. Define a bijective function $f: V(G) \rightarrow\{1,2, \ldots, n\}$ as follows

$f\left(v_{i}\right)=i$ for $1 \leq i \leq n$.

For any vertex $v_{i} \in V(G)$, if $i$ is odd

$$
\begin{aligned}
& \sum_{\nu_{i}} f(u)=\sum_{j=1}^{\frac{n-1}{2}} f\left(v_{2 j}\right)=\sum_{j=1}^{\frac{n-1}{2}} 2 j=2 \sum_{j=1}^{\frac{n-1}{2}} j \\
& =2\left(1+2+\cdots+\frac{n-1}{2}\right)
\end{aligned}
$$




$$
=\frac{n-1}{2}\left(\frac{n+1}{2}\right)=k_{1} \text { (say) }
$$

For any vertex $v_{i} \in V(G)$, if $i$ is even

$$
\begin{aligned}
\sum_{v_{i}} f(u \in E(G) & =\sum_{j=1}^{\frac{n-1}{2}} f\left(v_{2 j-1}\right)=\sum_{j=1}^{\frac{n-1}{2}}(2 j-1)=2 \sum_{j=1}^{\frac{n-1}{2}} j\left(\frac{n+1}{2}\right) \\
& =2\left(1+2+\cdots+\frac{n+1}{2}\right)-\left(\frac{n+1}{2}\right) \\
& =\frac{n+1}{2}\left(\frac{n+3}{2}\right)-\left(\frac{n+1}{2}\right) \\
& =\left(\frac{n+1}{2}\right)^{2}=k_{2} \text { (say) }
\end{aligned}
$$

Thus for any vertex $v \in V(G)$ we have two constants $k_{1}$ or $k_{2}$.

Case 2: When $n$ is even

The vertex set and edge set be defined as $V(G)=\left\{v_{1}, v_{2}, \ldots, v_{n}\right\}$. As per the definition of $G^{o}\left(P_{n}\right)$ we construct the edge set with respect to the index of the vertices as follows $E(G)=$ $\left\{v_{i} v_{2 j}: 1 \leq j \leq \frac{n}{2}\right.$ and for $i$ is odd $\} \cup\left\{v_{i} v_{2 j-1}: 1 \leq j \leq \frac{n}{2}\right.$ and for $i$ is even $\}$. Define a bijective function $f: V(G) \rightarrow\{1,2, \ldots, n\}$ as follows

$f\left(v_{i}\right)=i$ for $1 \leq i \leq n$.

For any vertex $v_{i} \in V(G)$, for $i$ is odd

$$
\begin{aligned}
\sum_{v_{i} u \in E(G)} f(u) & =\sum_{j=1}^{\frac{n}{2}} f\left(v_{2 j}\right)=\sum_{j=1}^{\frac{n}{2}} 2 j=2 \sum_{j=1}^{\frac{n}{2}} j \\
& =2\left(1+2+\cdots+\frac{n}{2}\right) \\
& =\frac{n}{2}\left(\frac{n}{2}+1\right)=k_{1} \text { (say) }
\end{aligned}
$$

For any vertex $v_{i} \in V(G)$, for $i$ is even

$$
\begin{aligned}
& \sum_{v_{i}} f(u)=\sum_{j=1}^{\frac{n}{2}} f\left(v_{2 j-1}\right)=\sum_{j=1}^{\frac{n}{2}} 2 j-1=2 \sum_{j=1}^{\frac{n}{2}} j-\left(\frac{n}{2}\right) \\
& =2\left(1+2+\cdots+\frac{n}{2}\right)-\left(\frac{n}{2}\right) \\
& =\frac{n}{2}\left(\frac{n}{2}+1\right)-\left(\frac{n}{2}\right) \\
& =\left(\frac{n}{2}\right)^{2}=k_{2} \text { (say) }
\end{aligned}
$$

Thus for any vertex $v \in V(G)$ we have two constants $k_{1}$ or $k_{2}$.

Hence the graph $G^{o}\left(P_{n}\right)$ has 1-vertex-bimagic vertex labeling. 


\section{1-vertex bimagic vertex labeling for complete symmetric multipartite graph}

Let $H_{n, p}, n>1$ and $p>1$ denote the complete symmetric multipartite graph with $p$ parts, each of which contains $n$ vertices. $H_{n, p}$ is a graph with $p$ partition which has equal number of vertices and every vertex of $G$ in each partition connected to all other vertices of the remaining $(p-1)$ partitions. The number of vertices and edges in $H_{n, p}$ is $n p$ and $\left(\begin{array}{l}p \\ 2\end{array}\right) n^{2}$ respectively. Another way of describing $H_{n . p}$ is as follows: Consider a complete graph $K_{p}$ with $p$ vertices $\left\{x_{1}, x_{2}, x_{3}, \ldots, x_{p}\right\}$. By replacing every vertex in $K_{p}$ with $n$ pairs of vertices, each joined to all vertices corresponding to the neighbours of the original vertex of $K_{p}$, we obtain the graph $H_{n, p}$.

Example 3.2. 1-vertex bimagic vertex labeling for complete symmetric multipartite graphs $H_{6,6}$ and $H_{5,6}$ is given below
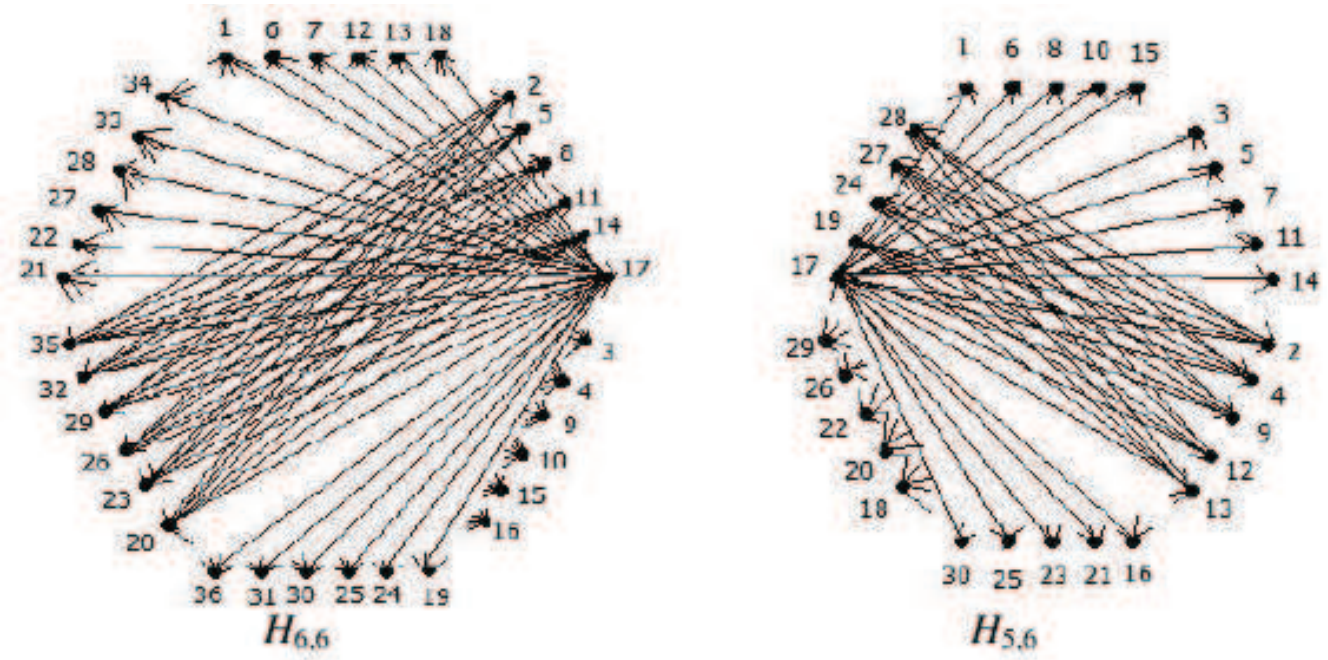

$H_{5.6}$

Figure 2: 1-vertex bimagic vertex labeling for complete symmetric multipartite graph

Theorem 3.7. Let $n>1$ and $p>1$. $H_{n, p}$ has a 1-vertex bimagic vertex labeling, for

(i) any $p$, when $n$ is even

(ii) even $p$, when $n$ is odd.

Proof. Consider a graph $H_{n, p}$. We prove this theorem in two cases.

Case 1: For any $p$, when $n$ is even

Let $x_{i j}$ be the vertices of $H_{n, p}, 1 \leq i \leq n ; 1 \leq j \leq p$. Label the vertices in the following way. Let $m= \begin{cases}\frac{p}{2} & \text { if } p \equiv 0 \bmod 2 \\ \frac{p+1}{2} & \text { otherwise }\end{cases}$ 
For $1 \leq j \leq m, f\left(x_{i, j}\right)= \begin{cases}j+(i-1) m & \text { if } i \text { is odd } \\ m-j+1+(i-1) m & \text { if } i \text { is even }\end{cases}$

For $m+1 \leq j \leq p, f\left(x_{i, j}\right)= \begin{cases}n m+(j-m)+(i-1) m & \text { if } i \text { is odd } \\ m(n+2)-j+1+(i-1) m & \text { if } i \text { is even }\end{cases}$

For $1 \leq j \leq m$, the sum of the labels of the $n$ vertices in each set $j$ is $\frac{n}{2}(m n+1)=S_{1}$ say. For $m+1 \leq j \leq p$, the sum of the labels of the $n$ vertices in each set $j$ is $\frac{n}{2}(n(m+p)+1)=S_{2}$ say.

Sub case (i): When $p$ is even

For any vertex $x \in V(G)$ in the interval $1 \leq j \leq m$

$$
\sum_{x y \in E(G)} f(y)=(m-1) S_{1}+m S_{2}=k_{1}
$$

and for any vertex $x \in V(G)$ in the interval $m+1 \leq j \leq p$

$$
\sum_{x y \in E(G)} f(y)=m S_{1}+(m-1) S_{2}=k_{2}
$$

Sub case (ii): When $p$ is odd

For any vertex $x \in V(G)$ in the interval $1 \leq j \leq m$

$$
\sum_{x y \in E(G)} f(y)=(m-1) S_{1}+(m-1) S_{2}=k_{1}
$$

and for any vertex $x \in V(G)$ in the interval $m+1 \leq j \leq p$

$$
\sum_{x y \in E(G)} f(y)=m S_{1}+(m-2) S_{2}=k_{2}
$$

Thus for any vertex $x \in V(G)$ we have two constants $k_{1}$ or $k_{2}$ for $n$ is even and $p$ is even or odd.

Case 2: If $p$ is even and $n$ is odd then we can prove this case in two sub cases. Let $m=\frac{p}{2}$.

Sub Case (i): when $m$ is odd then $2 t+1=m$. The labeling is defined below

$$
f\left(x_{i, j}\right)= \begin{cases}2 j-1, & 1 \leq j \leq t+1 \text { and } i=1, \\ 2(j-t-1), & t+2 \leq j \leq 2 t+1 \text { and } i=1 \\ 4 t+3-j, & 1 \leq j \leq 2 t+1 \text { and } i=2 \\ 5 t+4-j, & 1 \leq j \leq t+1 \text { and } i=3 \\ 7 t+5-j, & t+2 \leq j \leq 2 t+1 \text { and } i=3 \\ j+(i-1)(2 t+1), & 1 \leq j \leq 2 t+1 \text { and } i>3, i \text { even } \\ 2 t+2-j+(i-1)(2 t+1), & 1 \leq j \leq 2 t+1 \text { and } i>3, i \text { odd }\end{cases}
$$


and

$$
f\left(x_{i, 2 t+j+1}\right)= \begin{cases}n(2 t+1)+2 j-1, & 1 \leq j \leq t+1 \text { and } i=1, \\ n(2 t+1)+2(j-t-1), & t+2 \leq j \leq 2 t+1 \text { and } i=1, \\ n(2 t+1)+4 t+3-j, & 1 \leq j \leq 2 t+1 \text { and } i=2, \\ n(2 t+1)+5 t+4-j, & 1 \leq j \leq t+1 \text { and } i=3, \\ n(2 t+1)+7 t+5-j, & t+2 \leq j \leq 2 t+1 \text { and } i=3, \\ n(2 t+1)+j+(i-1)(2 t+1), & 1 \leq j \leq 2 t+1 \text { and } i>3, i \text { even, } \\ n(2 t+1)+2 t+2-j+(i-1)(2 t+1), & 1 \leq j \leq 2 t+1 \text { and } i>3, i \text { odd } .\end{cases}
$$

For every $j$ in the interval $1 \leq j \leq m$, we split the calculation for $j=1$ to $t+1$ and $j=t+2$ to $2 t+1$ as follows

for $j=1$ to $t+1, f\left(x_{1, j}\right)+f\left(x_{2, j}\right)+f\left(x_{3, j}\right)=2 j-1+4 t+3-j+5 t+4-j=9 t+6$

for $j=t+2$ to $2 t+1, f\left(x_{1, j}\right)+f\left(x_{2, j}\right)+f\left(x_{3, j}\right)=2(j-t-1)+4 t+3-j+7 t+5-j=9 t+6$ and for $i>1$, $f\left(x_{2 i, j}\right)+f\left(x_{2 i+1, j}\right)=j+(2 i-1)(2 t+1)+2 t+2-j+(2 i+1-1)(2 t+1)=(2 t+2)+(4 i-1)(2 t+1)$. Thus the sum of the labels of $n$ vertices in each set $j$ is $=f\left(x_{1, j}\right)+f\left(x_{2, j}\right)+f\left(x_{3, j}\right)+\sum_{i=2}^{\frac{n-1}{2}}\left[f\left(x_{2 i, j}\right)+f\left(x_{2 i+1, j}\right)\right]$ $=(9 t+6)+(n-3)(t+1)+\left(\frac{n^{2}-n-6}{2}\right)(2 t+1)=S_{1}$, say.

For every $j$ in the interval $m+1 \leq j \leq p$, we split the calculation for $j=1$ to $t+1$ and $j=t+2$ to $2 t+1$ as follows for $j=1$ to $t+1$,

$$
\begin{aligned}
& f\left(x_{1,2 t+j+1}\right)+f\left(x_{2,2 t+j+1}\right)+f\left(x_{3,2 t+j+1}\right) \\
& \quad=n(2 t+1)+2 j-1+n(2 t+1)+4 t+3-j+n(2 t+1)+5 t+4-j \\
& \quad=3 n(2 t+1)+9 t+6
\end{aligned}
$$

for $j=t+2$ to $2 t+1$,

$$
\begin{aligned}
f( & \left.x_{1,2 t+j+1}\right)+f\left(x_{2,2 t+j+1}\right)+f\left(x_{3,2 t+j+1}\right) \\
& =n(2 t+1)+2(j-t-t 1)+n(2 t+1)+4 t+3-j+n(2 t+1)+7 t+5-j \\
& =2 n(2 t+1)+9 t+6
\end{aligned}
$$

and for $i>1$,

$$
\begin{aligned}
f\left(x_{2 i, 2 t+j+1}\right)+f\left(x_{2 i+1,2 t+j+1}\right) & =n(2 t+1)+j+(2 i-1)(2 t+1)+n(2 t+1)+2 t+2-j+(2 i)(2 t+1) \\
& =2 n(2 t+1)+2 t+2+(4 i-1)(2 t+1) .
\end{aligned}
$$


Thus the sum of the labels of $n$ vertices in each set $j$ is $=f\left(x_{1,2 t+j+1}\right)+f\left(x_{2,2 t+j+1}\right)+f\left(x_{3,2 t+j+1}\right)+\sum_{i=2}^{\frac{n-1}{2}}\left[f\left(x_{2 i, 2 t+j+1}\right)+f\left(x_{2 i+1,2 t+j+1}\right)\right]$ $=[3 n(2 t+1)+(9 t+6)]+(n-3) n(2 t+1)+(n-3)(t+1)+\left(\frac{n^{2}-n-6}{2}\right)(2 t+1)=S_{2}$ say. For any vertex $x \in V(G)$ in the interval $1 \leq j \leq m$

$$
\sum_{x y \in E(G)} f(y)=(m-1) S_{1}+m S_{2}=k_{1}
$$

And for any vertex $x \in V(G)$ in the interval $m+1 \leq j \leq p$

$$
\sum_{x y \in E(G)} f(y)=m S_{1}+(m-1) S_{2}=k_{2}
$$

Thus in this case, for any vertex $x \in V(G)$ we have two constants $k_{1}$ or $k_{2}$.

Sub Case (ii): when $m$ is even then $2 t+1=m+1$. The labeling is defined below The labeling of $f\left(x_{i, j}\right)$ is same as given in above sub case (i) and the labeling of $f\left(x_{i, 2 t+j+1}\right)$ is shown below

$$
f\left(x_{i, 2 t+j+1}\right)= \begin{cases}n(2 t+1)+2 j-1, & 1 \leq j \leq t+1 \text { and } i=1, \\ n(2 t+1)+2(j-t), & t+1 \leq j \leq 2 t-1 \text { and } i=1, \\ n(2 t+1)+4(t-1)+3-j, & 1 \leq j \leq 2 t-1 \text { and } i=2, \\ n(2 t+1)+5(t-1)+4-j, & 1 \leq j \leq t+1 \text { and } i=3, \\ n(2 t+1)+7(t-1)+5-j, & t+1 \leq j \leq 2 t-1 \text { and } i=3, \\ n(2 t+1)+j+(i-1)(2 t-1), & 1 \leq j \leq 2 t-1 \text { and } i>3, i \text { even, } \\ n(2 t+1)+2(t-1)+2-j+(i-1)(2 t-1), & 1 \leq j \leq 2 t-1 \text { and } i>3, i \text { odd } .\end{cases}
$$

For every $j$ in the interval $1 \leq j \leq m+1$, we split the calculation for $j=1$ to $t+1$ and $j=t+2$ to $2 t+1$ as follows

for $j=1$ to $t+1, f\left(x_{1, j}\right)+f\left(x_{2, j}\right)+f\left(x_{3, j}\right)=2 j-1+4 t+3-j+5 t+4-j=9 t+6$

for $j=t+2$ to $2 t+1, f\left(x_{1, j}\right)+f\left(x_{2, j}\right)+f\left(x_{3, j}\right)=2(j-t-1)+4 t+3-j+7 t+5-j=9 t+6$

and for $i>1, f\left(x_{2 i, j}\right)+f\left(x_{2 i+1, j}\right)=j+(2 i-1)(2 t+1)+2 t+2-j+(2 i+1-1)(2 t+1)$

$=(2 t+2)+(4 i-1)(2 t+1)$

Thus the sum of the labels of $n$ vertices in each set $j$ is

$=f\left(x_{1, j}\right)+f\left(x_{2, j}\right)+f\left(x_{3, j}\right)+\sum_{i=2}^{\frac{n-1}{2}}\left[f\left(x_{2 i, j}\right)+f\left(x_{2 i+1, j}\right)\right]$

$=(9 t+6)+(n-3)(t+1)+\left(\frac{n^{2}-n-6}{2}\right)(2 t+1)=S_{1}$ say.

For every $j$ in the interval $m+2 \leq j \leq p$, we split the calculation for $j=1$ to $t$ and $j=t+1$ to $2 t-1$ as follows

for $j=1$ to $t$,

$$
f\left(x_{1,2 t+j+1}\right)+f\left(x_{2,2 t+j+1}\right)+f\left(x_{3,2 t+j+1}\right)
$$




$$
\begin{aligned}
& =n(2 t+1)+2 j-1+n(2 t+1)+4(t-1)+3-j+n(2 t+1)+5(t-1)+4-j \\
& =3 n(2 t+1)+9(t-1)+6
\end{aligned}
$$

for $j=t+2$ to $2 t-1$,

$$
\begin{aligned}
& f\left(x_{1,2 t+j+1}\right)+f\left(x_{2,2 t+j+1}\right)+f\left(x_{3,2 t+j+1}\right) \\
& \quad=n(2 t+1)+2(j-t)+n(2 t+1)+4(t-1)+3-j+n(2 t+1)+7(t-1)+5-j \\
& \quad=3 n(2 t+1)+9(t-1)+6
\end{aligned}
$$

and for $i>1$

$$
\begin{aligned}
f( & \left.x_{2 i, 2 t+j+1}\right)+f\left(x_{2 i+1,2 t+j+1}\right) \\
& =n(2 t+1)+j+(i-1)(2 t-1)+n(2 t+1)+2(t-1)+2-j+(i-1)(2 t-1) \\
& =2 n(2 t+1)+2(t-1)+2+(4 i-1)(2 t-1)
\end{aligned}
$$

Thus the sum of the labels of $n$ vertices in each set $j$ is

$=f\left(x_{1,2 t+j+1}\right)+f\left(x_{2,2 t+j+1}\right)+f\left(x_{3,2 t+j+1}\right)+\sum_{i=2}^{\frac{n-1}{2}}\left[f\left(x_{2 i, 2 t+j+1}\right)+f\left(x_{2 i+1,2 t+j+1}\right)\right]$

$=[3 n(2 t+1)+9(t-1)+6]+(n-3) n(2 t+1)+(n-3) t+\left(\frac{n^{2}-n-6}{2}\right)(2 t-1)=S_{2}$ say.

For any vertex $x \in V(G)$ in the interval $1 \leq j \leq m+1$

$$
\sum_{x y \in E(G)} f(y)=m S_{1}+(m-1) S_{2}=k_{1}
$$

And for any vertex $x \in V(G)$ in the interval $m+2 \leq j \leq p$

$$
\sum_{x y \in E(G)} f(y)=(m+1) S_{1}+(m-2) S_{2}=k_{2}
$$

Thus in this case, for any vertex $x \in V(G)$ we have two constants $k_{1}$ or $k_{2}$.

Hence using the above cases $H_{n, p}$ has a 1-vertex bimagic vertex labeling, if either $n$ is even or $p$ is even and $n$ is odd.

\section{General results on regular and bi-regular graphs}

All the graphs for which 1-vertex bimagic vertex labeling is done so far are either regular or bi-regular. But the Complete graph $K_{n}$ is not a 1-vertex bimagic, since it clearly admits distinct neibourhood sum for every vertex. Also form the definition and the theorems proved above we observe that the vertex set of every 1-vertex bimagic biregular graphs are partitioned into two sets $V_{1}$ and $V_{2}$ in such a way that each set contains the vertices of common count $k_{1}$ (degree $r_{1}$ ) or $k_{2}$ (degree $r_{2}$ ) respectively. We use this observation to prove the following theorems. 
Theorem 4.8. A regular or bi-regular graph $G$ has a 1-vertex-bimagic vertex labeling iff it has an odd 1-vertex-bimagic vertex labeling.

Proof. Suppose that $G$ is a 1 -vertex bimagic graph with $p$ vertices and $q$ edges. Then there exists a function $h: V(G) \rightarrow\{1,2, \ldots, p\}$ such that for every vertex $u, \sum_{u \nu \in E(G)} h(\nu)=k_{1}$ or $k_{2}$. Now we define $f: V(G) \rightarrow\{1,3, \ldots, 2 p-1\}$ such that $f\left(v_{i}\right)=2 h\left(v_{i}\right)-1 ; 1 \leq i \leq p$. For every vertex $u \in V(G)$ we have

$$
\begin{aligned}
\sum_{u v \in E(G)} f(v)=\sum_{u v \in E(G)}[2 h(v)-1] & =2 \sum_{u v \in E(G)} h(v)-\operatorname{deg}(u) \\
& =2\left(k_{1} \text { or } k_{2}\right)-\operatorname{deg}(u) \\
& =2 k_{1}-r_{1} \text { or } 2 k_{2}-r_{2}=s_{1} \text { or } s_{2} .
\end{aligned}
$$

Then $G$ has an odd 1-vertex-bimagic vertex labeling with common counts $s_{1}$ and $s_{2}$. Conversely, suppose that $G$ is a graph with $p$ vertices and $q$ edges and $f: V(G) \rightarrow\{1,3, \ldots, 2 p-1\}$ is an odd 1-vertex-bimagic vertex labeling with two common count $s_{1}$ and $s_{2}$. Then $h: V(G) \rightarrow$ $\{1,2, \ldots, p\}$ defined by $h\left(v_{i}\right)=\frac{1}{2}\left[f\left(v_{i}\right)+1\right]$ for $1 \leq i \leq p$.

For every vertex $u \in V(G)$ we have

$$
\begin{aligned}
\sum_{u v \in E(G)} h(v)=\sum_{u \nu \in E(G)} \frac{1}{2}[f(v)+1] & =\frac{1}{2}\left(\sum_{u v \in E(G)} f(v)+\sum_{u v \in E(G)} 1\right) \\
& =\frac{1}{2}\left(s_{1} \text { or } s_{2}\right)+\frac{1}{2} \operatorname{deg}(u)=k_{1} \text { or } k_{2}
\end{aligned}
$$

Hence 1-vertex-bimagic vertex labeling has two common counts $k_{1}=\frac{1}{2}\left(s_{1}+r_{1}\right)$ and $k_{2}=\frac{1}{2}\left(s_{2}+\right.$ $\left.r_{2}\right)$.

Theorem 4.9. A regular or bi-regular graph $G$ has a 1-vertex-bimagic vertex labeling iff it has an even 1-vertex-bimagic vertex labeling.

Proof. Suppose that $G$ is a 1-vertex-bimagic graph with $p$ vertices and $q$ edges. Then there exists a function $h: V(G) \rightarrow\{1,2, \ldots, p\}$ such that for every vertex $u, \sum_{u \nu \in E(G)} h(\nu)=k_{1}$ or $k_{2}$. Now we define $g: V(G) \rightarrow\{0,2, \ldots, 2 p-2\}$ such that $g\left(v_{i}\right)=2 h\left(v_{i}\right)-2 ; 1 \leq i \leq p$. For every vertex $u \in V(G)$ we have

$$
\begin{aligned}
\sum_{u v \in E(G)} g(\nu)=\sum_{u v \in E(G)}[2 h(\nu)-2] & =2 \sum_{u v \in E(G)} h(\nu)-2 \operatorname{deg}(u)=2\left(\left(k_{1} \text { or } k_{2}\right)-\operatorname{deg}(u)\right) \\
& =2 k_{1}-r_{1} \text { or } 2 k_{2}-r_{2}=s_{1} \text { or } s_{2}
\end{aligned}
$$

Then $G$ has an even 1-vertex bimagic vertex labeling with common counts $s_{1}$ and $s_{2}$. 
Conversely, suppose that $G$ is a graph with $p$ vertices and $q$ edges and $g: V(G) \rightarrow\{0,2, \ldots$, $2 p-2\}$ is an even 1-vertex-bimagic vertex labeling with two common edge count $s_{1}$ and $s_{2}$. Then $h: V(G) \rightarrow\{1,2, \ldots, p\}$ defined by $h\left(v_{i}\right)=\frac{1}{2}\left[g\left(v_{i}\right)+2\right]$ for $1 \leq i \leq p$.

For every vertex $u \in V(G)$ we have

$$
\begin{aligned}
\sum_{u v \in E(G)} h(\nu)=\sum_{u v \in E(G)} \frac{1}{2}[g(v)+2] & =\frac{1}{2}\left(\sum_{u v \in E(G)} g(v)+\sum_{u v \in E(G)} 2\right) \\
& =\frac{1}{2}\left(s_{1} \text { or } s_{2}\right)+\operatorname{deg}(u)=k_{1} \text { or } k_{2}
\end{aligned}
$$

Hence 1-vertex-bimagic vertex labeling has two common counts $k_{1}=\frac{1}{2}\left(s_{1}\right)+\operatorname{deg}(u)$ and $k_{2}=$ $\frac{1}{2}\left(s_{2}\right)+\operatorname{deg}(u)$.

Theorem 4.10. If $H$ is a regular or bi-regular 1-vertex bimagic graph then $G=H_{n}^{m K_{1}}(m>1)$, has a 1-vertex bimagic vertex labeling.

Proof. Consider a graph $H$ is a 1 -vertex bimagic with $n$ vertices $\left\{x_{1}, x_{2}, x_{3}, \ldots, x_{n}\right\}$. By replacing every vertex in $H$ with $m$ isolated vertices, each joined to all vertices corresponding to the neighbours of the original vertex of $H$, we obtain the graph $G=H_{n}^{m K_{1}}$ which is a multipartite graph. For $1 \leq i \leq m$ and $1 \leq j \leq n$, Let $x_{i j}$ be the vertices of $G$ that replace $x_{j}, 1 \leq j \leq n$ in $H$. Given that $H$ has a 1-vertex bimagic vertex labeling, then there exist a function $f: V \rightarrow$ $\{1,2, \ldots, n\}$ such that $\sum_{u n \in E(H)} f(v)=k_{1}$ or $k_{2}$ where $k_{1}$ and $k_{2}$ are constants. Let $V_{1}$ be the set of vertices having constant value $k_{1}$, that is for every vertex $y \in V_{1}, \sum_{y x \in E(H)} f(x)=k_{1}$. Let $V_{2}$ be the set of vertices having constant value $k_{2}$, that is for every vertex $y \in V_{2}, \sum_{y x \in E(H)} f(x)=k_{2}$.

Label the vertices of $G$ in the following way $g\left(x_{i, j}\right)=f\left(x_{j}\right)+(i-1) n$ for $1 \leq i \leq m$ and $1 \leq j \leq n$.

For every $j$ in the interval $1 \leq j \leq n$,

$$
\sum_{i=1}^{m} g\left(x_{i, j}\right)=\sum_{i=1}^{m}\left[f\left(x_{j}\right)+(i-1) n\right]=\sum_{i=1}^{m} f\left(x_{j}\right)+\sum_{i=1}^{m}(i-1) n=m f\left(x_{j}\right)+\frac{n m(m-1)}{2}
$$

Let $U$ and $W$ be the two partitions of the graph $G$ in such a way that $U$ contains the sets which is having the vertices of $V_{1}$ in $H$ and $W$ contains the sets which is having the vertices of $V_{2}$ in $H$.

For any vertex $u \in U$, the $\operatorname{deg}(u)=r_{1}$ (say).

$$
\sum_{u x_{i, j} \in E(G)} g\left(x_{i, j}\right)=\sum_{u x_{i, j} \in E(G)} \sum_{i=1}^{m} g\left(x_{i, j}\right)=\sum_{u x_{i, j} \in E(G)}\left(m f\left(x_{j}\right)+\frac{n m(m-1)}{2}\right)
$$




$$
\begin{aligned}
& =\sum_{u x_{i, j} \in E(G)} m f\left(x_{j}\right)+\sum_{u x_{i, j} \in E(G)}\left(\frac{n m(m-1)}{2}\right) \\
& =m k_{1}+\left(\frac{n m(m-1)}{2}\right) \operatorname{deg}(u)=m k_{1}+\left(\frac{n m(m-1)}{2}\right) r_{1}=s_{1} \text { (say) }
\end{aligned}
$$

For any vertex $u \in W$, the $\operatorname{deg}(u)=r_{2}$ (say).

$$
\begin{aligned}
\sum_{u x_{i, j} \in E(G)} g\left(x_{i, j}\right) & =\sum_{u x_{i, j} \in E(G)} \sum_{i=1}^{m} g\left(x_{i, j}\right)=\sum_{u x_{i, j} \in E(G)}\left(m f\left(x_{j}\right)+\frac{n m(m-1)}{2}\right) \\
& =\sum_{u x_{i, j} \in E(G)} m f\left(x_{j}\right)+\sum_{u x_{i, j} \in E(G)}\left(\frac{n m(m-1)}{2}\right) \\
& =m k_{2}+\left(\frac{n m(m-1)}{2}\right) \operatorname{deg}(u)=m k_{2}+\left(\frac{n m(m-1)}{2}\right) r_{2}=s_{2} \text { (say) }
\end{aligned}
$$

Thus for any vertex $u \in V(G)$ we have two constants $s_{1}$ or $s_{2}$.

Hence $G=H_{n}^{m K_{1}}$ has a 1-vertex bimagic vertex labeling.

\section{Conclusion}

Theorem 3.7 shows a 1-vertex bimagic vertex labeling for certain class of symmetric multipartite graphs and the Theorem 4.10 shows a 1-vertex bimagic vertex labeling for a class of multipartite graphs. We belive that complete multipartite graph and multipartite graph have 1-vertex bimagic vertex labeling along with some condition. So, we conclude this paper with the following open problems.

\section{Open Problem 5.1}

(i) Does there exist 1-vertex bimagic vertex labeling for other classes of multipartite graphs.

(ii) Are there any necessary and sufficient conditions for a complete multipartite graph to have a 1-vertex bimagic vertex labeling.

\section{References}

[1] J. B. Babujee, On edge bimagic labeling, Journal of Combinatorics, Information \& System Sciences, 28-29, No. 1-4 (2004), 239-244.

[2] J. B. Babujee, Bimagic labeling in path graphs, The Mathematics Education, 38 No. 1 (2004), 12-16.

[3] J. A. Gallian, A Dynamic Survey of Graph Labeling, Electronic Journal of Combinatorics, 17 (2010), \# DS6.

[4] J.A. MacDougall, M. Miller, Slamin and W.D. Wallis, Vertex-magic total labelings of Graphs, Utilitas Math., 61 (2002), 3-21.

[5] Mirka Miller, Chris Rodger, Rinovia Simanjuntak, Distance magic labeling of Graphs, Australasian Journal Of Combinatorics, 28 (2003), 305-315.

[6] S. B. Rao, Sigma Graphs: A survey, Labelings of Discrete Structures and Applications, Editors: B.D. Acharya, S. Arumugam, A. Rosa, Copyright (2008), Narosa Publishing House, New Delhi, India. 
[7] J. Sedlacek, Problem 27 in Theory of graphs and its applications, Proc Symposium Smolenice 1963, Prague (1964), 163-164.

[8] V. Swaminathan and P. Jeyanthi, ( $a, d)$-1-vertex antimagic vertex labeling, Utilitas Math., 74 (2007), 179-186.

[9] V. Vilfred, Perfectly regular graphs or cyclic regular graphs and $\Sigma$-labeling and partition, Srinivasa Ramanujan Celebrating Int. Conf. in Mathematics, Anna University, Madras, India, Abstract, A23.

[10] V. Vilfred, Sigma partition and sigma labeled graphs, Journal of Decision and Mathematical Sciences, 10, No. 1-3 (2005).

[11] V. Vilfred, $\Sigma$-labeled graphs and circulant graphs, Ph.D. Thesis, University of Kerala, Trivandrum, India (1994).

Department of Mathematics, Anna University, Chennai-600 025, India.

E-mail: baskarbabujee@yahoo.com

Department of Mathematics, Anna University, Chennai-600 025, India.

E-mail: babi_mit@yahoo.co.in 